\title{
Cinobufacini Ameliorates Dextran Sulfate Sodium-Induced Colitis in Mice through Inhibiting M1 Macrophage Polarization
}

\author{
Si-wei Wang, Yong-feng Bai, Yuan-yuan Weng, Xue-yu Fan, Hui Huang, Fang Zheng, \\ Yi Xu, and Feng Zhang \\ Department of Core Facility (S.W., Fa.Z., Fe.Z.), Clinical Laboratory (Y.-B., Y.-W., X.-F., H.H., Fe. Z.), and Department of Urology \\ (Y.X.), People's Hospital of Quzhou, Quzhou, People's Republic of China
}

Received October 23, 2018; accepted December 31, 2018

\begin{abstract}
Cinobufacini is a traditional Chinese medicine used clinically that has antitumor and anti-inflammatory effects. It improves colitis outcomes in the clinical setting, but the mechanism underlying its function yet to be uncovered. We investigated the protective effects and mechanisms of cinobufacini on colitis using a dextran sulfate sodium (DSS)-induced colitis mouse model, mainly focusing on the impact of macrophage polarization. Our results showed that cinobufacini dramatically ameliorated DSS-induced colitis in mice. Cinobufacini treatment reduced the infiltration of activated $\mathrm{F} 4 / 80^{+}$and/or $\mathrm{CD}^{+} 8^{+}$macrophages into the colon in DSS-induced colitis mice. More importantly, cinobufacini significantly decreased the quantity of $\mathrm{M} 1$ macrophages and the expression of
\end{abstract}

proinflammatory cytokines such as interleukin-6, tumor necrosis factor $\alpha$, and inducible nitric oxide synthase. Cinobufacini also increased the population of M2 macrophages and the expression of anti-inflammatory factors such as interleukin-10 and arginase-1 in DSS-induced colitis mice. Furthermore, our study demonstrated that cinobufacini inhibited M1 macrophage polarization in lipopolysaccharide-induced RAW 264.7 cells. Mechanistically, our in vivo and in vitro results showed that cinobufacini inhibition of $\mathrm{M} 1$ macrophage polarization may be associated with the suppression of nuclear factor $\kappa \mathrm{B}$ activation. Our study suggests that cinobufacini could ameliorate DSS-induced colitis in mice by inhibiting M1 macrophage polarization.

\section{Introduction}

Inflammatory bowel disease (IBD), which mainly includes ulcerative colitis and Crohn's disease, is a complex, multifactorial disease. In past decades, extensive studies have been conducted on IBD and its pathogenesis, but its etiology remains unclear. A variety of mouse colitis models have been developed as indispensable tools for deciphering the pathogenesis of IBD and validating potential treatments in preclinical settings. The dextran sulfate sodium (DSS)-induced colitis mouse model has been widely used for human ulcerative colitis research due to its rapidity, simplicity, reproducibility, and controllability (Chassaing et al., 2014). DSS is type of water-soluble, negatively charged, sulfated polysaccharide with a highly variable molecular mass, ranging from 5 to $1400 \mathrm{kDa}$. Acute, chronic, and relapsing models of intestinal inflammation can be achieved by modifying the concentration and frequency of DSS administration (Chassaing et al., 2014). The most severe murine colitis, which most closely resembles

This work was supported by Zhejiang Provincial Natural Science Foundation [LYY18H280001]; The Chinese Medicine Science Foundation of Zhejiang Province [2018ZB134] and Quzhou Technology Projects [2016J016, 20172004]. https://doi.org/10.1124/jpet.118.254516. human ulcerative colitis, can be induced by administration of 40-50 kDa DSS in drinking water (Okayasu et al., 1990).

Macrophages play an important role in regulating the immune response. Divergency in the cellular microenvironment influences the phenotypes and function of macrophages and induces various immunologic responses (Wynn and Vannella, 2016). Macrophages, which can be activated in a number of ways, can be categorized as two main groups, designated M1 and M2. M1 macrophages promote inflammation, and M2 macrophages inhibit inflammation (Sica and Mantovani, 2012). Increasing evidence has suggested that regulating macrophage activity and polarization could be an effective approach for treating ulcerative colitis (Zhang et al., 2014; Eissa et al., 2017; Abron et al., 2018; Li et al., 2018).

Toad glandular secretions and skin extractions contain various natural agents that may provide a unique resource for novel drug development. Dried secretions of the auricular and skin glands of Chinese toad (Bufo bufo gargarizans), referred to as Chansu, have been widely used for hundreds of years in traditional Chinese medicine (TCM) for treating infection, inflammation, heart disease, and cancer (Qi et al., 2014). Cinobufacini (Huachansu), a sterilized hot water extraction of dried toad skin, was developed as a drug to treat hepatitis B virus and several types of cancers (Qi et al., 2014). The components of cinobufacini were

ABBREVIATIONS: Arg-1, arginase-1; DAI, disease activity index; DSS, dextran sulfate sodium; IBD, inflammatory bowel disease; IKK, inhibitory factor- $\kappa$ B kinase; IL-6, interleukin-6; IL-10, interleukin-10; iNOS, inducible nitric oxide synthase; LPS, lipopolysaccharide; NF- $\kappa$ B, nuclear factor $\kappa$ B; $\mathrm{PCR}$, polymerase chain reaction; qRT-PCR, quantitative reverse-transcription polymerase chain reaction; TCM, traditional Chinese medicine; TNF$\alpha$, tumor necrosis factor $\alpha$. 


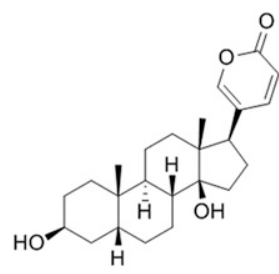

Bufalin

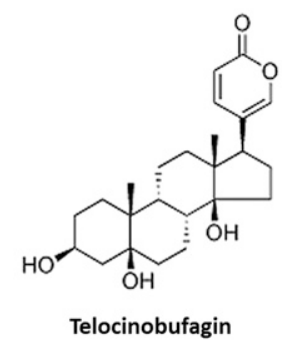

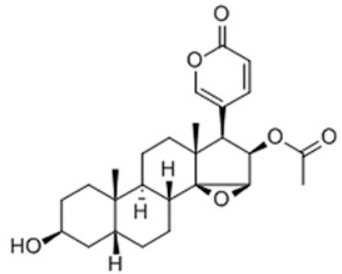

Cinobufagin

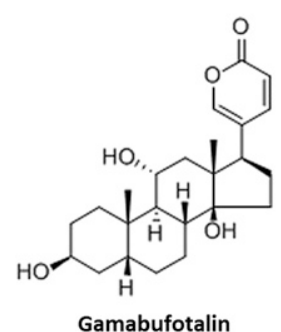

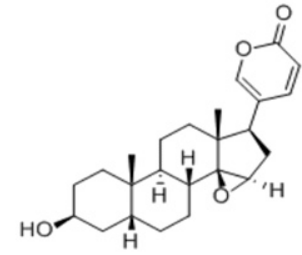

Resibufogenin

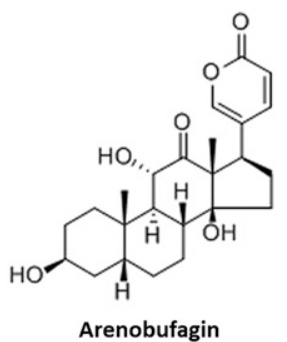

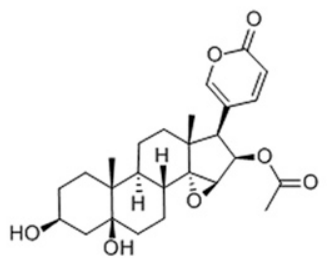

Cinobufotalin

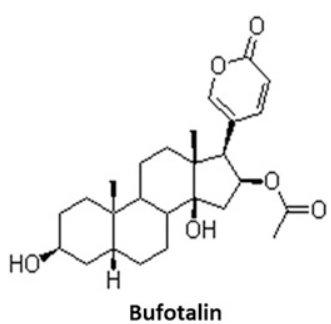

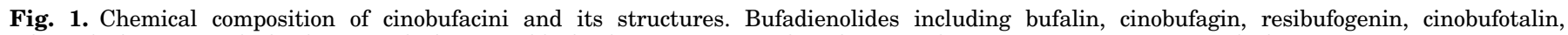
telocinobufagin, gamabufotalin, arenobufagin, and bufotalin are recognized as the major bioactive components in cinobufacini.

analyzed in a previous study using liquid chromatography with mass spectrometry (Wu et al., 2012), and bufadienolidesincluding bufalin, cinobufagin, resibufogenin, cinobufotalin, telocinobufagin, gamabufotalin, arenobufagin, and bufotalin-were found to be the major bioactive components (Fig. 1). However, the capacity of cinobufacini to alleviate acute colitis and its underlying mechanisms is unclear.

Existing studies have suggested that the anti-inflammation and anticancer potential of cinobufacini lies in targeting the nuclear factor $\kappa \mathrm{B}(\mathrm{NF}-\kappa \mathrm{B})$ signaling pathway, which is a crucial hallmark of inflammation and cancer in various experimental models (Qi et al., 2014; Xie et al., 2016). NF- $\kappa$ B is an important transcription factor that regulates macrophage phenotypic polarization (Porta et al., 2009; Tugal et al., 2013). In this study, we thus evaluated the clinical function of cinobufacini treatment of colitis using a DSS-induced colitis mouse model, and we investigated its effects and molecular mechanism on macrophage polarization in vivo and in vitro. The results shed new light on the mechanism and development of TCM as clinical drug to treat colitis in humans.

\section{Materials and Methods}

Reagents and Antibodies. Cinobufacini capsules (National Drug Standard: Z20050846, the purity of bufadienolides are approximately 30\%), with pharmacologic constituents listed in Table 1, were purchased from Shanxi Dongtai Pharmaceutical (Xianyang, People's Republic of China) for in vivo animal experiments. The cinobufacini injections (National Drug Standard: Z34020273), with pharmacologic constituents listed in Table 2, were purchased from Anhui Jinchan Biochemical (Huaibei, People's Republic of China) and were used for in vitro cell experiments. Dextran sulfate sodium (DSS) was purchased from MP Biomedicals (cat. no. 160110; Santa Ana, CA). Antibodies against F4/80 (cat. no. 2370; Cell Signaling Technology, Beverly, MA), CD68 (cat. no. ab125212; Abcam, Cambridge, MA), CD32 (cat. no. sc-166711; Santa Cruz Biotechnology, Dallas, TX), TNF- $\alpha$ (cat. no. sc-52746; Santa Cruz), CD206 (cat. no. ab8918; Abcam), NF- $\kappa$ B p65 (cat. no. 8242; Cell Signaling Technology), $\operatorname{IKK} \beta$ (cat. no. 2370; Cell Signaling Technology), phospho-inhibitory factor- $\kappa$ B kinase (IKK) $\alpha / \beta$ (cat. no. 2697; Cell Signaling Technology), $\mathrm{I} \kappa \mathrm{B} \alpha$ (cat. no. 4812; Cell Signaling Technology), phospho-I $\kappa \mathrm{B} \alpha$ (cat. no. 2859; Cell Signaling Technology), and $\beta$-actin (cat. no. A1978; MilliporeSigma, Darmstadt, Germany) were used in this study.

Experimental Animals. Male ICR mice (6 weeks of age) were obtained from SLAC Laboratory Animals (Shanghai, People's Republic of China) and housed at the Zhejiang Chinese Medical University Laboratory Animal Research Center. All mice were handled in accordance with U.S. National Institutes of Health animal care guidelines. The protocol was previously approved by the Animal Care and Use Committee of Quzhou People's Hospital.

Establishment of DSS-Induced Colitis Mouse Model and Treatment. To study the clinical efficiency of cinobufacini treatment in a DSS-induced colitis mouse model, as shown in Fig. 2A, 36 mice were randomly assigned to three groups with 12 animals per group. 1) In the control group, mice drank blank distilled water every day without DSS and received intragastrically administrated distilled water from days 1 to 10.2 ) In the DSS group, mice drank distilled water with 5\% DSS for 7 days to induce acute colitis and received intragastrically administered distilled water from days 1 to 10. 3) In the DSS + cinobufacini group, mice drank water with 5\% DSS from days 1 to 7 and were treated with $100 \mathrm{mg} / \mathrm{kg}$ per day of intragastrically administered cinobufacini from days 1 to 10 .

Clinical Scoring and Histologic Analysis. The disease activity index (DAI), comprising body weight, stool consistency, and stool bleeding, was recorded daily, where DAI = Comprehensive score of (Body loss rate + Stool consistency + Stool bleeding)/3. Each score was determined as follows. Weight loss was scored as $0=$ none;

TABLE 1

Eight bufadienolides contained in a cinobufacini capsule

\begin{tabular}{lc}
\hline \multicolumn{1}{c}{ Ingredient } & Content (\%) \\
\hline Cinobufotalin & 2.03 \\
Cinobufagin & 0.66 \\
Resibufogenin & 0.35 \\
Telocinobufagin & 5.63 \\
Gamabufotalin & 42.90 \\
Arenobufagin & 40.53 \\
Bufalin & 0.94 \\
Bufotalin & 6.95 \\
\hline
\end{tabular}


TABLE 2

Eight bufadienolides contained in a cinobufacini injection

\begin{tabular}{lr}
\hline \multicolumn{1}{c}{ Ingredient } & Content \\
\hline & $n g / m l$ \\
Cinobufotalin & 4.43 \\
Cinobufagin & 2.04 \\
Resibufogenin & 1.31 \\
Telocinobufagin & 9.84 \\
Gamabufotalin & 102.15 \\
Arenobufagin & 107.37 \\
Bufalin & 1.55 \\
Bufotalin & 12.27 \\
\hline
\end{tabular}

$1=1 \%-5 \% ; 2=5 \%-10 \% ; 3=10 \%-15 \%$; and $4=>15 \%$. Stool consistency was scored as $0=$ normal; $1-2=$ loose stool; and $3-4=$ diarrhea. Stool bleeding was scored as $0=$ negative; $1=+; 2=++$; $3=+++$; and $4=++++$ (Jin et al., 2017).
For colon length measurements, all mice were sacrificed, and the colons were excised from the vermiform appendix to the anus at the end of experiment. The colon length was measured between the cecum and proximal rectum.

For the histopathologic evaluation, the colon tissues were fixed in $10 \%$ formalin and stained with H\&E. The histopathologic scores were determined according to the criteria described by Wang et al. (2017). Mean scores were assessed by calculating five different fields at $400 \times$ magnification by two pathologists who were blinded to the sample groups.

Cell Culture. The RAW 264.7 cell line was obtained from the Shanghai Bank of Cell Lines (Shanghai, People's Republic of China) and was cultured in RPMI-1640 (HyClone Laboratories, Logan, UT) containing $10 \%$ fetal bovine serum (BBI Life Sciences Corporation, Shanghai, People's Republic of China), $100 \mathrm{U} / \mathrm{ml}$ penicillin, and $100 \mathrm{U} / \mathrm{ml}$ streptomycin. The cell line was grown at $37^{\circ} \mathrm{C}$ in a humidified incubator with $5 \% \mathrm{CO}_{2}$.

Flow Cytometry. RAW 264.7 cells were pretreated with dimethylsulfoxide, lipopolysaccharide (LPS) $(1 \mu \mathrm{g} / \mathrm{ml})$, or LPS
A

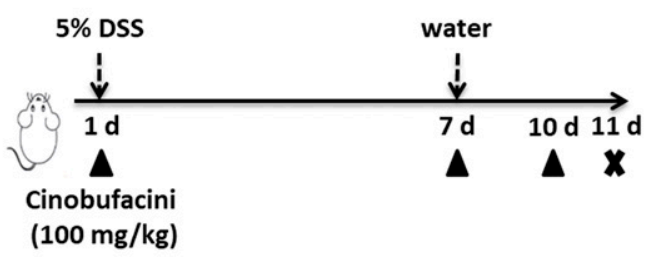

C

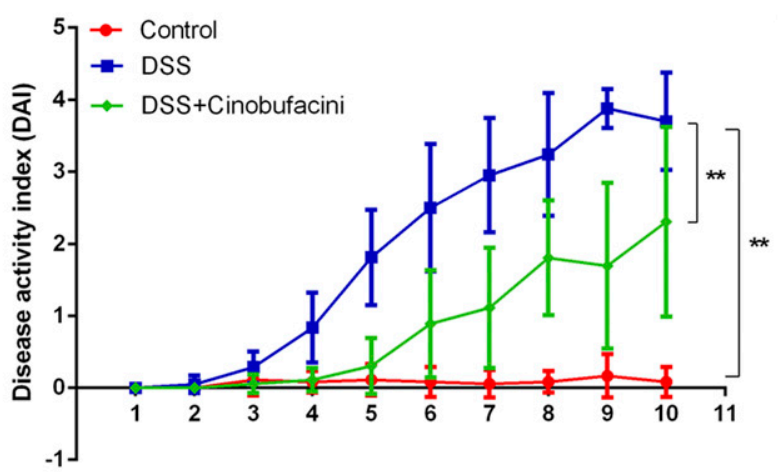

B
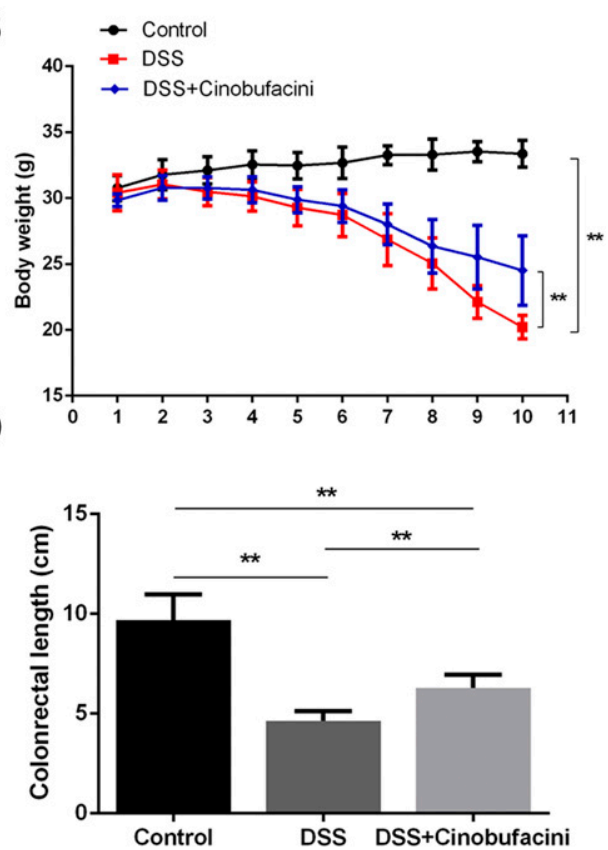

E
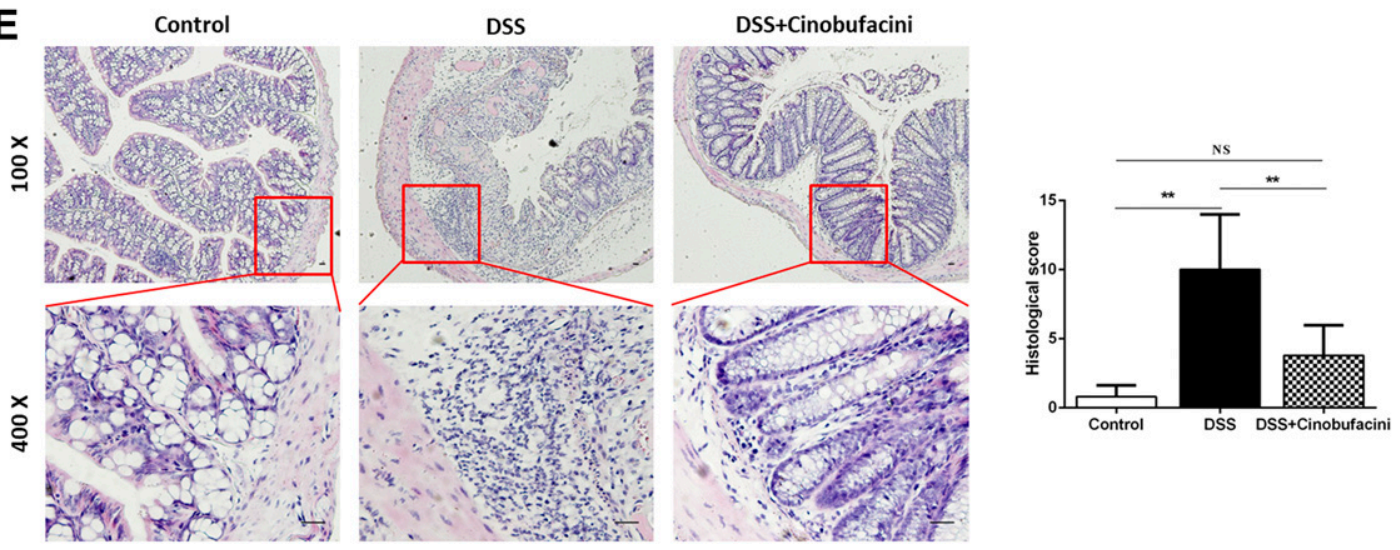

Fig. 2. Cinobufacini attenuates DSS-induced acute colitis in mice. Male ICR mice were given $5 \%$ DSS in drinking water (ad libitum) for 7 days to induce acute colitis. Cinobufacini $(100 \mathrm{mg} / \mathrm{kg})$ was administered for 10 days during DSS treatment via oral gavage once per day. Mice were sacrificed at day 10 after colitis induction. (A) The schematic diagram for DSS-induced colitis and cinobufacini administration. (B) Body weight change during the experimental period. (C) Disease activity index (DAI) during the disease process. (D) The lengths of colons from each group of mice. Values are expressed as mean \pm S.D. $(n=12$ for each group). (E) Representative images of H\&E staining of colon tissue and histopathologic injury score from each group. Scale bar: $200 \mu \mathrm{m} . * P<0.05 ; * * P<0.01 ; \mathrm{NS}$, not statistically significant. 
TABLE 3

Primers used for real-time PCR

\begin{tabular}{lll}
\hline Description & \multicolumn{1}{c}{ Sense primer $\left(5^{\prime} \rightarrow 3^{\prime}\right)$} & \multicolumn{1}{c}{ Antisense primer $\left(5^{\prime} \rightarrow 3^{\prime}\right)$} \\
\hline$C D 16$ & AATGCACACTCTGGAAGCCAA & CACTCTGCCTGTCTGCAAAAG \\
$I L-6$ & CTGCAAGAGACTTCCATCCAG & AGTGGTATAGACAGGTCTGTTGG \\
$T N F-\alpha$ & CTGAACTTCGGGGTGATCGG & GGCTTGTCACTCGAATTTGAGA \\
$i N O S$ & GTTCTCAGCCCAACAATACAAGA & GTGGACGGGTCGATGTCAC \\
$C D 206$ & CTCTGTTCAGCTATTGGACGC & TGGCACTCCCAAACATAATTTGA \\
$I L-10$ & CTTACTGACTGGCATGAGGATCA & GCAGCTCTAGGAGCATGTGG \\
$A r g-1$ & CTCCAAGCCAAAGTCCTTAGAG & GGAGCTGTCATTAGGGACATCA \\
$G A P D H$ & TGAGGCCGGTGCTGAGTATGT & CAGTCTTCTGGGTGGCAGTGAT \\
\hline
\end{tabular}

Arg-1, arginase-1; GAPDH, glyceraldehyde-3-phosphate dehydrogenase; IL, interleukin; iNOS, inducible nitric oxide synthase; TNF- $\alpha$, tumor necrosis factor $\alpha$.

$(1 \mu \mathrm{g} / \mathrm{mL})+$ cinobufacini $(50 \mathrm{mg} / \mathrm{ml})$ for 24 hours. For the fluorescenceactivated cell sorter (FACS) analysis, the pretreated RAW 264.7 cells were harvested and washed with cold phosphate-buffered saline twice, then stained with fluorescein isothiocyanate-conjugated anti-CD16/32 (1:1000; BioLegend, San Diego, CA) and phycoerythrin-conjugated antiCD206 (1:1000; BioLegend) for 15 minutes at room temperature in the dark. The stained cells were analyzed by flow cytometry (FC-500; Beckman Coulter, Brea, CA).

Immunohistochemical Staining and Analysis. Paraffinembedded sections were deparaffinized, rehydrated, and followed by antigen retrieval according to standard protocols as reported elsewhere (Wang et al., 2018). After that, the sections were treated with $3 \% \mathrm{H}_{2} \mathrm{O}_{2}$ and incubated with blocking goat serum for 1 hour at $37^{\circ} \mathrm{C}$. Sections were incubated with primary antibody overnight at $4^{\circ} \mathrm{C}$, followed by using MaxVision HRP-Polymer anti-Rabbit IHC Kit (MXB Biotechnologies, Fuzhou, People's Republic of China).

The immunohistochemical evaluation was conducted as described by Mashimo et al. (2014). In short, the staining intensity of the colon tissue section is defined by four grades, expressed as an integer from 0 to 3 . The proportion of immunohistochemically stained positive cells
A
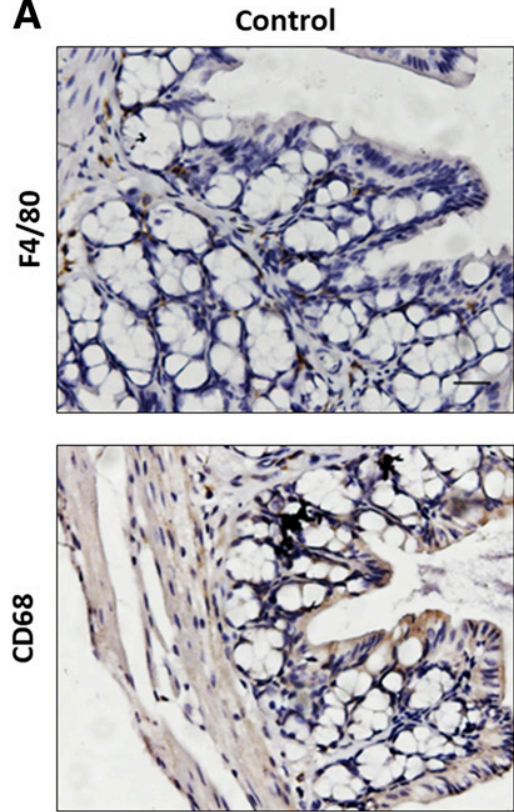
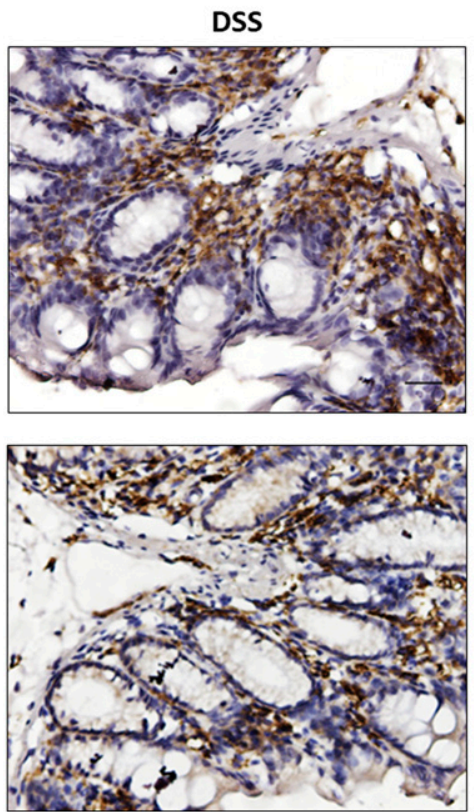

C

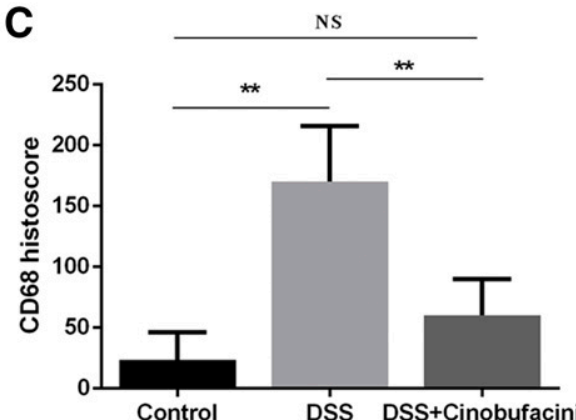

Fig. 3. Cinobufacini suppresses the activation of macrophages in DSS-induced colitis mice. (A) Expression of F4/80 and CD68 in the colon evaluated using immunohistochemistry. Representative images of F4/80 and CD68 expression in mouse colon are shown. Scale bar: $200 \mu \mathrm{m}$. (B and C) Histoscore of F4/80 and CD68 expression. The histoscore was calculated by multiplying the staining intensity value and the percentage of positive cells. Values are expressed as mean \pm S.D. ${ }^{* *} P<0.01 ; \mathrm{NS}$, not statistically significant. 
A
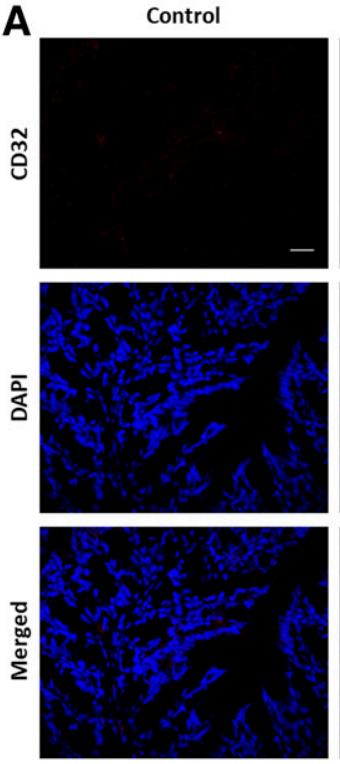

B

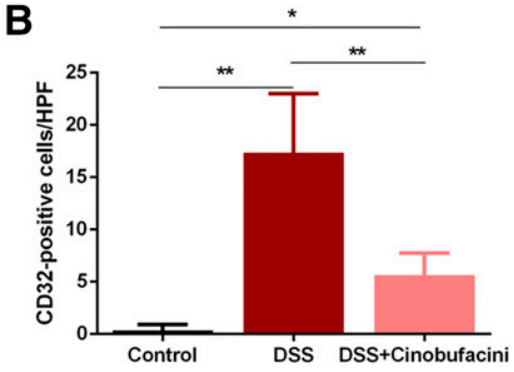

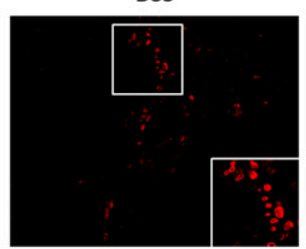
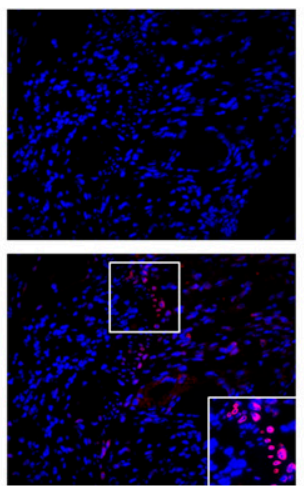

DSS DSS+Cinobufacin
DSS+Cinobufacini
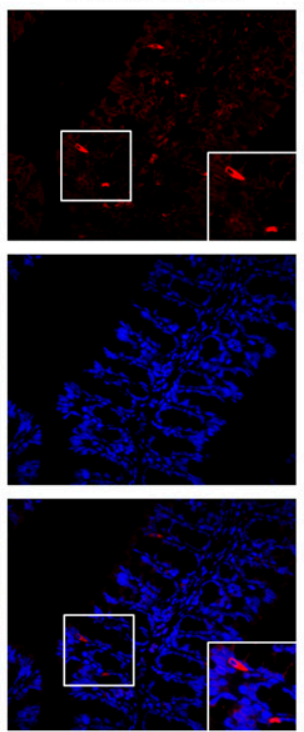

D

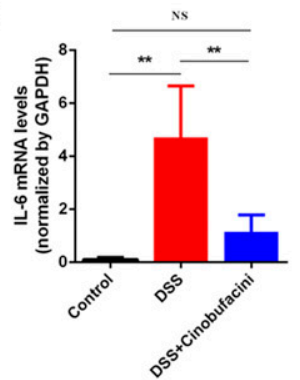

Control

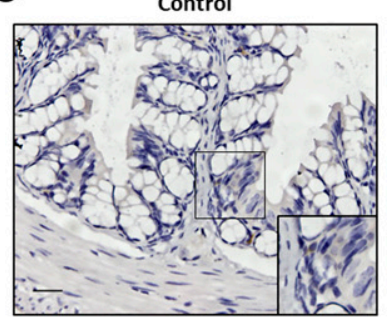

DSS+Cinobufacini

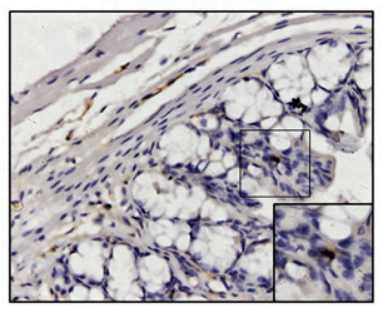

DSS
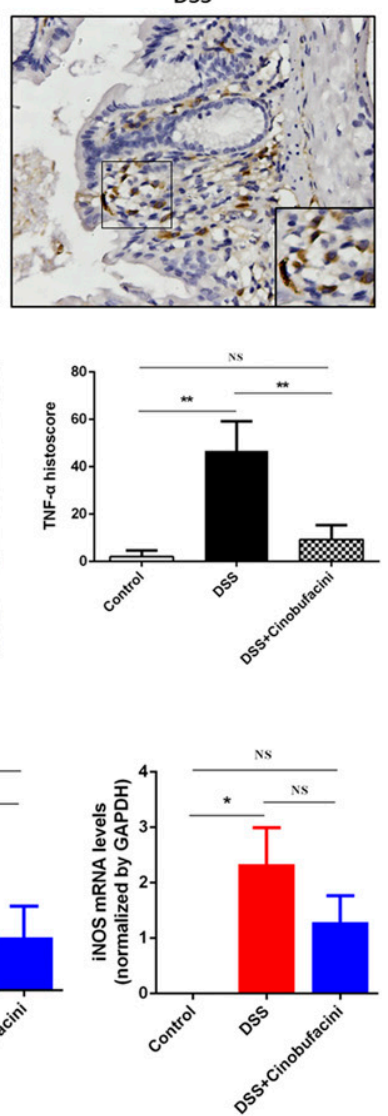

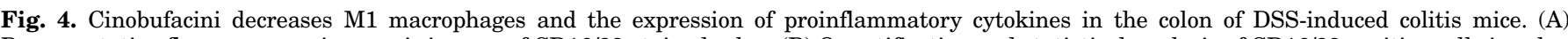

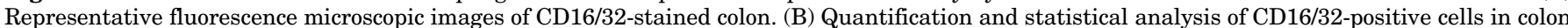

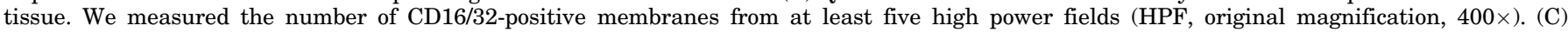

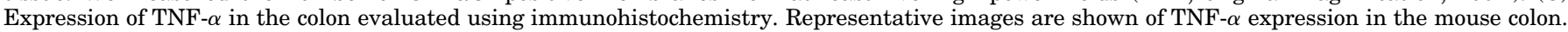

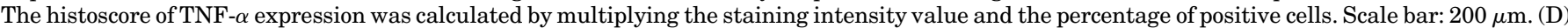

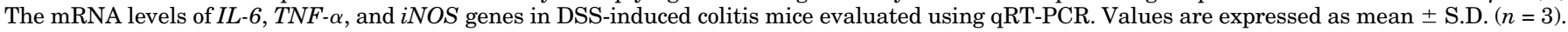
$* P<0.05 ; * * P<0.01 ;$ NS, not statistically significant.

is expressed as a value between $0 \%$ and $100 \%$. These two values (intensity and the percentage of positive cells) are then multiplied to obtain histologic scores (range: 0-300), which we used for further comparative analysis. The scores of stained colon tissue sections were accessed by two independent pathologists. A total of five fields at $400 \times$ magnification were assessed for each sample. The final count represented the mean of the histoscore from these five slides.

Immunofluorescence Staining. RAW 264.7 cells were grown on glass cover slides. After treatment, the cells were fixed in $4 \%$ paraformaldehyde and permeabilized in $0.2 \%$ Triton X-100 for 15 minutes. For paraffin-embedded tissues, the slides were deparaffinized with xylene, dehydrated in decreasing concentrations of ethanol, and then permeabilized. Immunostaining was performed with primary antibody in $5 \%$ normal goat serum at $4^{\circ} \mathrm{C}$ overnight. The sections were washed with cold phosphate-buffered saline and incubated with Alexa Fluor 488/594-labeled secondary antibody at room temperature for 1 hour. The images were examined by fluorescence microscopy (Eclipse Ti-S; Nikon, Tokyo, Japan).

Quantitative Reverse-Transcription Polymerase Chain Reaction. For the quantitative reverse-transcription polymerase chain reaction (qRT-PCR) analysis, the colonic tissue and RAW 264.7 cells were homogenized, and RNA was extracted using TRIzol reagent (Tiangen Biotech, Beijing, People's Republic of China) according to the manufacturer's protocol. Briefly, $40 \mathrm{mg}$ of colonic tissues of each sample or one well of RAW 264.7 cells from a six-well plate were lysed using $1 \mathrm{ml}$ of TRIzol reagent. For full homogenization, the colon tissue was homogenized in a mechanical homogenizer after the addition of TRIzol reagent until no large tissue mass was observed. To remove residual DSS contaminants, a lithium chloride method was used to remove all polysaccharides including DSS, as described by Viennois et al. (2018).

The cDNA was synthesized with reverse transcriptase kits (Thermo Fisher Scientific, Waltham, MA). Real-time polymerase chain reaction (PCR) was performed using SYBR Green (Sangon Biotech, Shanghai, People's Republic of China), and data were acquired in a LightCycler 480 instrument (Roche, Basel, Switzerland) and analyzed using the comparative cycle threshold method. The primers are listed in Table 3. Target-gene transcription of each sample was normalized to the respective levels of glyceraldehyde-3-phosphate dehydrogenase (GAPDH).

Western Blotting. RAW 264.7 cells were grown in six-well plates. After treatment, the cells were lysed using radioimmunoprecipitation assay buffer (50 mM Tris-HCl, pH 7.4, 2 mM EDTA, $150 \mathrm{mM} \mathrm{NaCl}$, $0.1 \%$ sodium dodecyl sulfate, and $1 \%$ NP-40) containing protease inhibitor cocktail (Roche) and phosphatase inhibitor cocktail (1 mM sodium orthovanadate, $5 \mathrm{mM}$ sodium fluoride, $3 \mathrm{mM} \beta$-glycerophosphate, and $4 \mathrm{mM}$ sodium tartrate). The lysates were centrifuged at $16,000 \mathrm{~g}$ for 10 minutes at $4^{\circ} \mathrm{C}$, and the supernatants were collected.

The protein concentration was determined using Nano-100 microscope spectrophotometer (Allsheng Instruments, Hangzhou, People's Republic China). Equal amounts of protein were separated by $10 \%$ SDSPAGE and transferred onto polyvinylidene difluoride membranes 
A
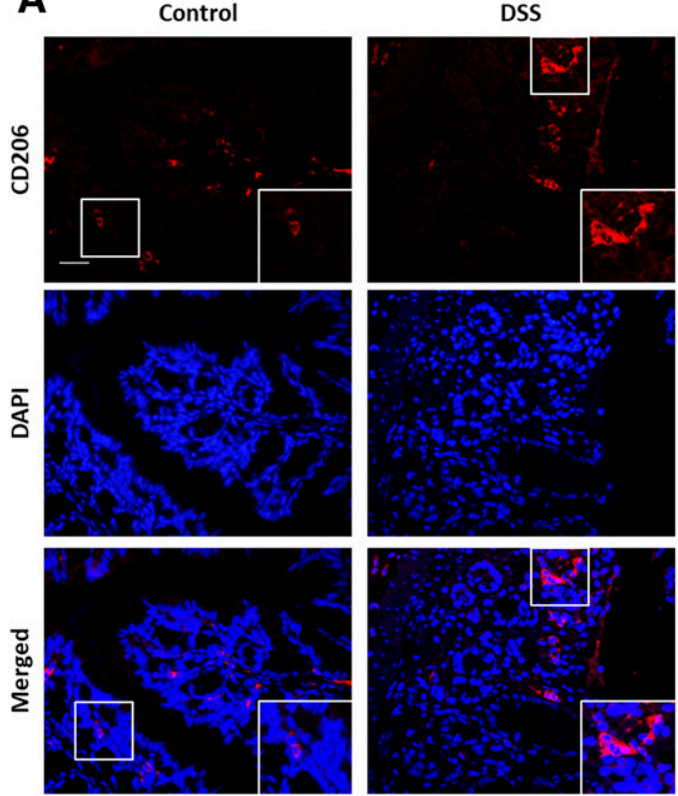

DSS+Cinobufacini
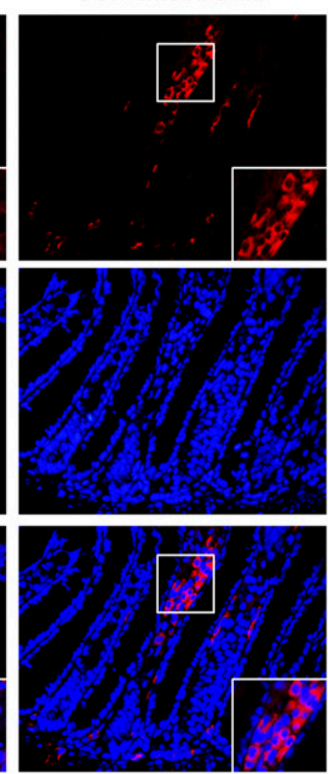

B

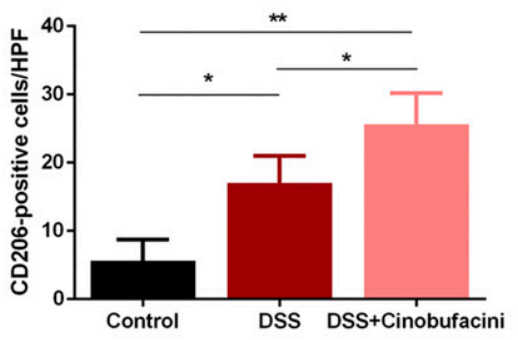

C

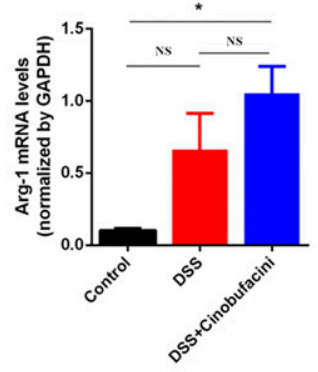

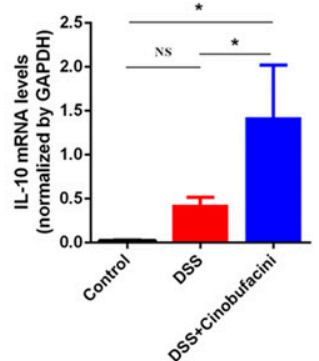

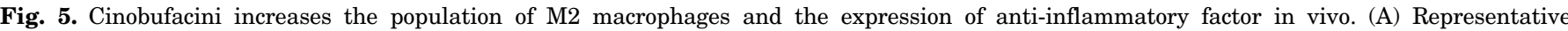

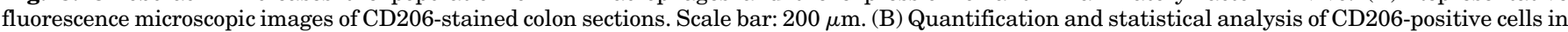

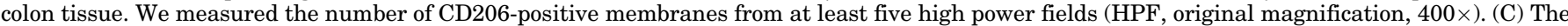

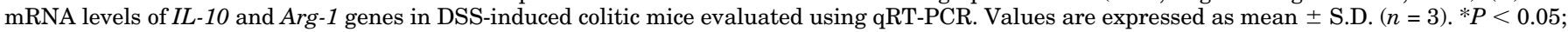
$* * P<0.01 ;$ NS, not statistically significant.

(Millipore, Darmstadt, Germany) according to standard methods. After blocking with $1 \%$ casein in Tris-buffered saline/Tween 20 for 1 hour at room temperature, the membranes were incubated with primer antibody at $4^{\circ} \mathrm{C}$ overnight. Anti-rabbit or anti-mouse IgG conjugated to horseradish peroxidase (Cell Signaling Technology) were used as secondary antibodies and incubated for 1 hour at room temperature.

Immune complexes were detected by the Tanon $4200 \mathrm{SF}$ system from Tanon Biotechnology (Shanghai, People's Republic of China). Band intensity was quantified using ImageJ software (U.S. National Institutes of Health, Bethesda, MD).

Statistical Analysis. Prism 5.0 (GraphPad Software, La Jolla, CA) was used for all statistical analyses. Data were expressed as mean \pm S.D. Differences among groups were assessed using one-way analysis of variance. Differences were considered to be statistically significant at $P<0.05$ and highly significant at $P<0.01$.

\section{Results}

Cinobufacini Attenuates DSS-Induced Acute Colitis in Mice. To estimate the clinical effect of cinobufacini on acute colitis, we established a DSS-induced colitis mouse model through providing ICR mice with drinking water containing $5 \%$ DSS for 7 days (Fig. 2A). The mice administered DSS had significant body weight loss compared with the control group. However, cinobufacini $(100 \mathrm{mg} / \mathrm{kg})$ treatment in the DSS-induced colitis mice significantly alleviated the loss of body weight compared with the mice in the DSS-only group (Fig. 2B). In addition, the cumulative DAI score, which indicated the severity of the disease, decreased obviously in the DDS + cinobufacini treatment group compared with the DSS-only group (Fig. 2C).

DSS administration resulted in remarkable shortening of the colon; cinobufacini administration significantly ameliorated the phenomenon (Fig. 2D). The histologic examination of mouse colon tissues showed that DSS induced a marked inflammatory response, characterized by a large number of inflammatory monocytes and macrophages infiltrating the colon, disruption of the architecture of colonic mucosa, and thickening of the lamina propria. The administration of cinobufacini significantly improved the pathologic changes in the DSS-induced colitis mice, as indicated by the reduced histologic injury score in the DSS + cinobufacini treatment group compared with the DSS-only group (Fig. 2E). Comprehensively, these results indicate that cinobufacini attenuated the severity of colitis in DSS-induced colitis mice.

Cinobufacini Suppresses the Infiltration of Activated Macrophages in DSS-Induced Colitis Mice. The infiltration of activated macrophages plays a crucial role in the development and perpetuation of intestinal inflammation (Zhu et al., 2016). To assess whether cinobufacini treatment in DSS-induced colitis in mice was agonistic to the infiltration of macrophages in the colon, we examined the macrophage infiltration by immunohistochemical staining of the macrophage markers F4/80 and CD68. The results showed a much higher number of $\mathrm{F} 4 / 80^{+}$or $\mathrm{CD}^{+} 8^{+}$macrophages in DSS group compared with the control group (Fig. 3). However, the number of activated $\mathrm{F} 4 / 80^{+}$or $\mathrm{CD} 68^{+}$macrophages in colon was markedly reduced by cinobufacini treatment (Fig. 3). These results suggest that cinobufacini treatment suppresses the infiltration of activated macrophages in the colon of DSSinduced colitis mice.

Cinobufacini Decreases M1 Macrophages and the Expression of Proinflammatory Cytokines in the Colon of DSS-Induced Colitis Mice. M1 and M2, two major subtypes of macrophages, have distinct functions in inflammatory response ( $\mathrm{Li}$ et al., 2018). M1 macrophages are considered to promote inflammation, and M2 macrophages function oppositely (Moore et al., 2013). CD16/32, tumor necrosis factor $\alpha$ (TNF- $\alpha$ ), interleukin 6 (IL-6), and induced 
A

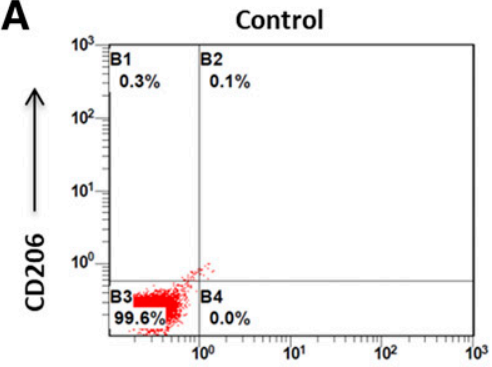

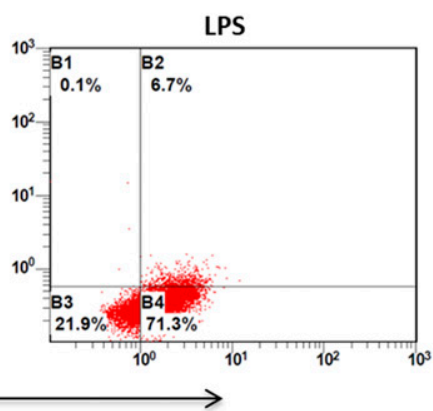

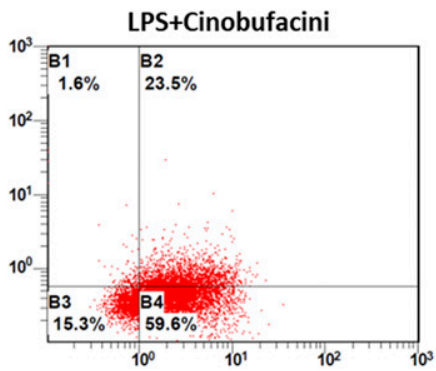

B
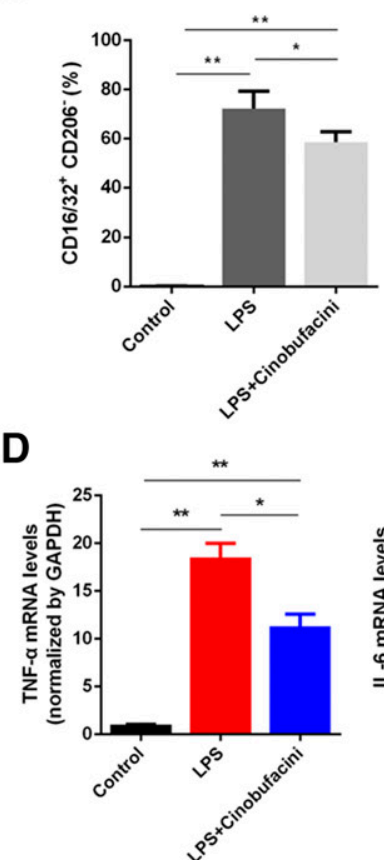

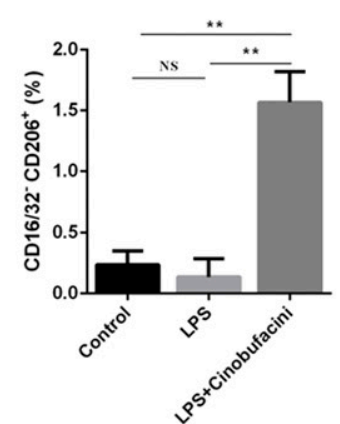

C
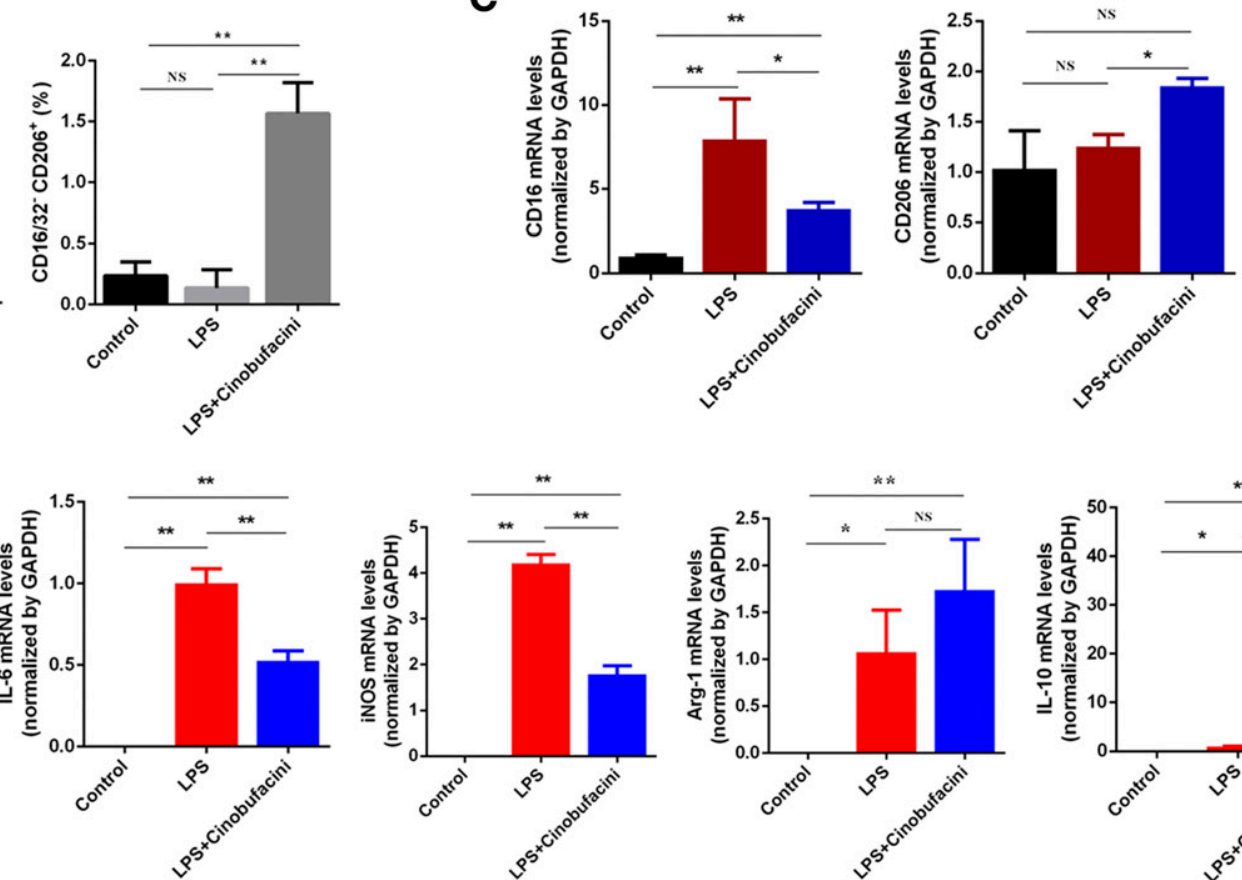

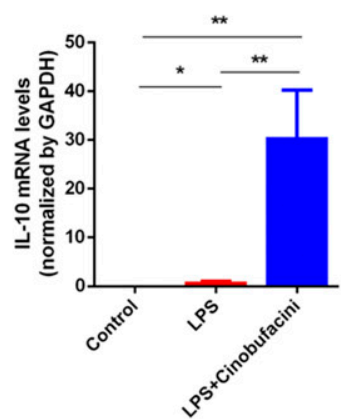

Fig. 6. Cinobufacini influences macrophage polarization and expression of inflammatory cytokines in LPS-induced RAW 264.7 macrophage cell line. RAW 264.7 cells were pretreated with dimethylsulfoxide, LPS $(1 \mu \mathrm{g} / \mathrm{ml})$, or LPS $(1 \mu \mathrm{g} / \mathrm{ml})+$ cinobufacini (50 mg/ml) for 24 hours. (A) Scatterplot of flow cytometry detection of M1 and M2 macrophages based on surface markers CD16/32 and CD206. (B) Percentage of CD16/32/CD206 ${ }^{-}$cells (M1) and CD206 ${ }^{+} / \mathrm{CD} 16 / 32^{-}$cells (M2) determined by flow cytometry. (C) M1 and M2 macrophage surface markers of CD16 and CD206 mRNA levels in LPSinduced RAW 264.7 cells evaluated using qRT-PCR. (D) Proinflammatory factors $I L-6, T N F-\alpha$, and $i N O S$ and anti-inflammatory factors $I L-10$ and Arg-1 determined by qRT-PCR. Values are expressed as mean \pm S.D. $(n=3) .{ }^{*} P<0.05 ; * * P<0.01$; NS, not statistically significant.

nitric oxide synthase (iNOS) are commonly used as markers for detecting M1 macrophages (Feng et al., 2014; Jiang et al., 2015). To investigate whether cinobufacini could affect the subpopulation of macrophages, we examined the number of M1 macrophages and the expression of proinflammatory cytokines associated with M1 phenotype in DSS-induced colitic mice.

Our results showed that DSS increased M1 macrophages in the mouse colon, as detected by immunofluorescence staining with antibodies against the M1 macrophage marker CD32. As shown in Fig. 4, A and B, a lower number of CD32-positive M1 cells was observed in the healthy control mice and cinobufacini treatment DSS-induced colitis mice. Similarly, the immunohistochemical staining results displayed that cinobufacini significantly inhibited TNF- $\alpha$ expression in the colon of DSSinduced colitis mice (Fig. 4C). In addition, the mRNA expression levels of the M1 macrophage marker genes $T N F-\alpha, I L-6$, and $i N O S$ in the colon decreased after cinobufacini treatment as detected by qRT-PCR (Fig. 4D).
Cinobufacini Increases the Population of M2 Macrophages and the Expression of Anti-inflammatory Factors In Vivo. Additionally, we evaluated the effect of cinobufacini on the activation of M2 macrophages in DSSinduced colitis mice. We used CD206 as a marker for M2 macrophages (Zhang et al., 2018). The immunofluorescence results showed that the number of CD206-positive cells in the colon were significantly increased after cinobufacini treatment in DSS-induced colitis mice (Fig. 5, A and B). The results of qRT-PCR also confirmed that cinobufacini could increase the expression of $I L-10$ and arginase-1 (Arg-1) expression in colon, which are mainly secreted by M2 macrophages (Zhang et al., 2018) (Fig. 5C). Taken together, these results revealed that cinobufacini could increase the M2 and decrease the M1 macrophage populations in DSS-induced colitis mice.

Cinobufacini Influences Macrophage Polarization and Expression of Inflammatory Cytokines in LPSInduced RAW 264.7 Cells. We used the RAW264.7 macrophage cell line as an in vitro model to study the effect of 
A
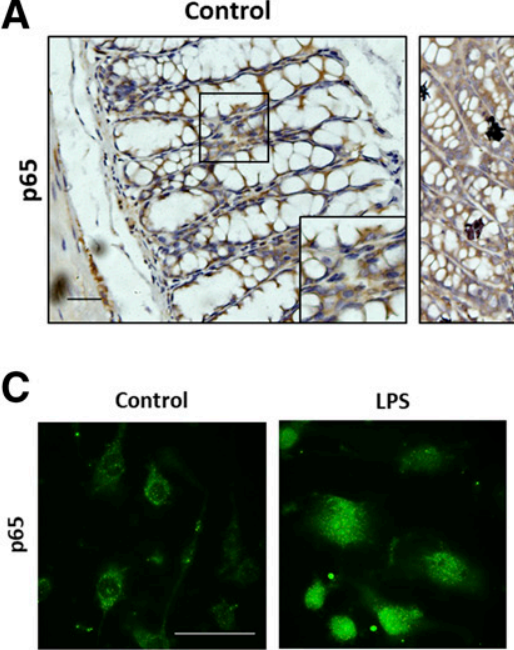

DSS
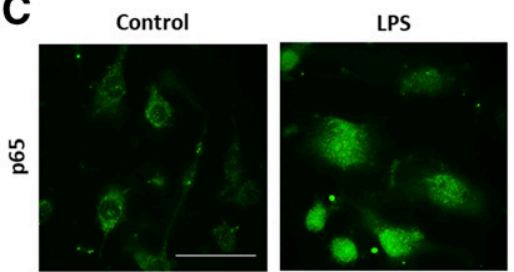

LPS+Cinobufacini
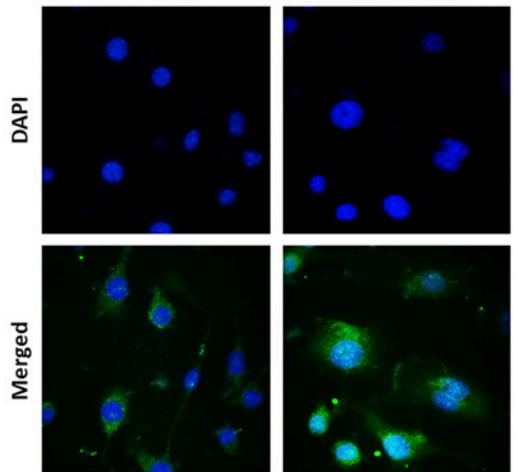
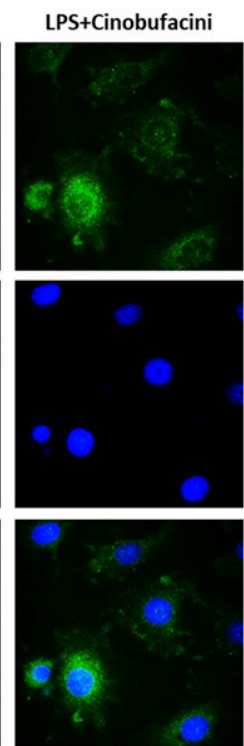

DSS+Cinobufacin

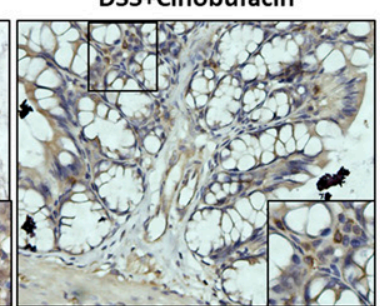

B

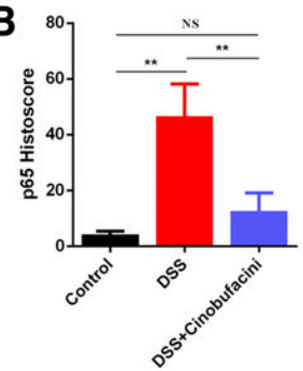

D

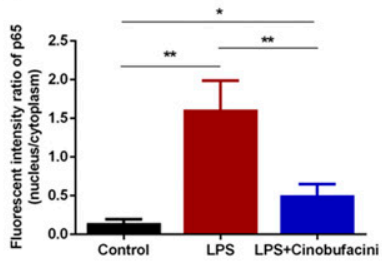

E

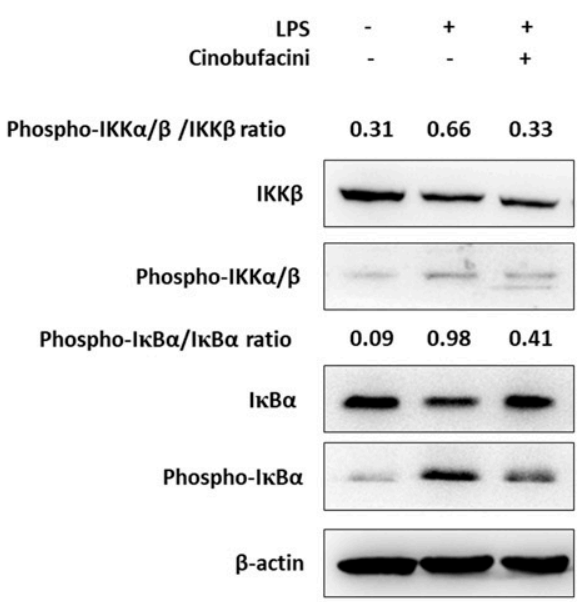

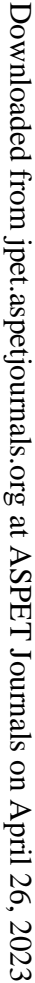

Fig. 7. Cinobufacini suppresses NF- $\kappa \mathrm{B}$ activation in DSS-induced colitis mice and LPS-induced RAW 264.7 cells. (A) Expression of NF- $\kappa \mathrm{B}$ p65 in the colon evaluated using immunohistochemistry. Representative images are shown of NF- $\kappa \mathrm{B}$ p65 expression in the mouse colon. Scale bar: $200 \mu \mathrm{m}$. (B) Histoscore of NF- $\kappa \mathrm{B}$ p65 expression calculated by multiplying the staining intensity value and the percentage of positive cells. (C) RAW 264.7 cells pretreated with dimethylsulfoxide, LPS $(1 \mu \mathrm{g} / \mathrm{ml})$, or LPS $(1 \mu \mathrm{g} / \mathrm{ml})+$ cinobufacini $(50 \mathrm{mg} / \mathrm{ml})$ for 24 hours with immunofluorescence staining of NF- $\kappa \mathrm{B}$ p65 in LPS-induced RAW264.7 cells. (D) Quantification of the fluorescent intensity of NF- $\kappa \mathrm{B}$ p65 in the nucleus relative to that of the cytoplasm. Scale bar: $200 \mu \mathrm{m}$. (E) Levels of IKK $\beta$, phospho-IKK $\alpha / \beta, \mathrm{I} \kappa \mathrm{B} \alpha$, and phospho-I $\kappa \mathrm{B} \alpha$ protein in LPS-induced RAW264.7 cells determined using Western blot analysis. ${ }^{*} P<0.05 ; * * P<0.01 ; \mathrm{NS}$, not statistically significant.

cinobufacini on the subpopulation of macrophages. As shown in Fig. 6, A and B, LPS stimulation only induced the RAW 264.7 cells to polarize into $\mathrm{CD} 206^{-} \mathrm{CD} 16 / 32^{+} \mathrm{M} 1$ macrophages. However, after cinobufacini + LPS treatment, a significant fraction of RAW264.7 cells differentiated into the CD206 ${ }^{+}$ CD16/32 $2^{-}$2 subtype, although the absolute proportion in the total number of cells was not very high (Fig. 6, A and B).

The populations of macrophages under LPS with or without cinobufacini treatment were further confirmed by examination of CD206 and CD32 mRNA expression using real-time qRT-PCR (Fig. 6C). M1 macrophages expressed and secreted $T N F-\alpha, I L-6$, and $i N O S$, whereas M2 macrophages expressed $I L-10$ and Arg-1. The qRT-PCR results showed that cinobufacini markedly inhibited the expression of $T N F-\alpha, I L-6$, and iNOS while increasing the expression of $I L-10$ and Arg-1 in RAW264.7 cells after treatment (Fig. 6D).

Cinobufacini Suppresses NF-אB Activation in DSSInduced Colitis Mice and LPS-Stimulated RAW 264.7 Cells. The canonical NF- $\kappa$ B pathway has been known as an important transcription factor regulating macrophage polarization (Kühnemuth et al., 2015). Because we observed in RAW 264.7 cells that cinobufacini promotes macrophage polarization in vitro, we investigated the activation of the NF- $\kappa$ B pathway in colon tissue of the DSS-induced colitis mice and in RAW264.7 cells with or without cinobufacini treatment.

As shown in Fig. 7, A and B, cinobufacini significantly suppressed NF- $\kappa \mathrm{B}$ p65 expression in mouse colon tissue by immunohistochemical staining. Nuclear translocation is a feature of NF- $\kappa \mathrm{B}$ activation. The immunofluorescence analysis revealed that cinobufacini treatment inhibited NF- $\kappa \mathrm{B}$ p65 accumulation in the nuclei of LPS-stimulated RAW264.7 cells (Fig. 7, C and D). As major regulatory components of the activity of NF- $\kappa \mathrm{B}$, the phosphorylation of $\mathrm{I} \kappa \mathrm{B} \alpha$ and $\mathrm{IKK} \alpha / \beta$ protein was reduced by cinobufacini treatment in LPSstimulated RAW264.7 cells (Fig. 7E). These results imply that cinobufacini regulates macrophage polarization through regulating the $\mathrm{NF}-\kappa \mathrm{B}$ activation pathway.

\section{Discussion}

In this study, we explored the effect and underlying mechanism of cinobufacini (toad skin extract) in treating colitis. We used a powerful DSS-induced colitis mouse model to study the clinical outcome after the drug treatment and studied the potential molecular targets. Our findings deepened our understanding of the anti-inflammatory activity of cinobufacini, owing to its inhibition of macrophage infiltration 


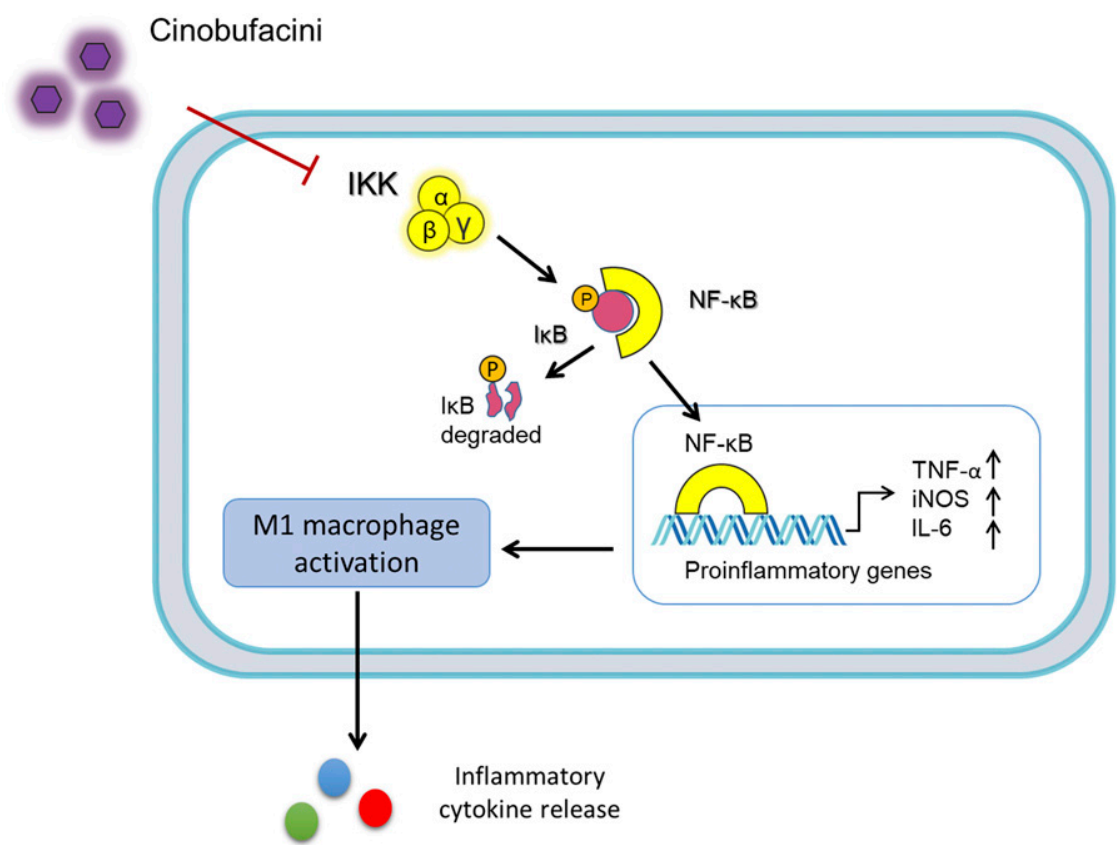

Fig. 8. Schematic diagram of cinobufacini-derived anti-inflammatory effect in the DSS-induced colitis model. and phenotypic polarization via suppression of the $\mathrm{NF}-\kappa \mathrm{B}$ pathway.

Cinobufacini, a TCM preparation with antitumor and antiinflammation activity, has a good record of clinical efficacy and safety (Meng et al., 2012; Zhang et al., 2017a). In the practice of TCM therapeutics, cinobufacini is also commonly used as a drug to treat acute and chronic colitis, but no clinical or experimental research has been officially reported. In the present study, we provide evidence that cinobufacini has an inhibitory effect on DSS-induced colitis in a mouse model.

Cinobufacini treatment prevented body weight loss by DSSinduced colitis. The DAI score also was much lower in cinobufacini-treated colitic mice, indicating an improved clinical outcome in colitis. The colon lengths and histopathologic findings showed improvement as well. All these results suggest that cinobufacini attenuates the clinical activity of DSS-induced colitis in mice.

However, cinobufacini is a complex compound that includes at least eight bufadienolides (Wu et al., 2012). It is unclear which of these ingredients exerts the major effect on inhibition of colitis, and it is also unknown whether these components have synergistic effects. Therefore, this remains an open question for further exploration in the future.

Recent studies have shown that a strong correlation between dysfunction of macrophages and the development of ulcerative colitis (Zhang et al., 2017b; Abron et al., 2018; Gan et al., 2018). Here, we proved that cinobufacini suppressed the infiltration of activated macrophages in DSS-induced colitis mice. Of note, our results suggest that cinobufacini could influence the polarization of macrophages, and thus affect the subtypes of macrophages with distinct characteristics of humoral factor production and gene expression (Kono et al., 2014). M1 macrophages, which express the phenotypic marker CD16/32 and proinflammatory cytokines (Mantovani et al., 2002; Koh et al., 2018), promote the development of colorectal inflammation. By contrast, M2 macrophages, which express CD206 and anti-inflammatory cytokines (Li et al., 2018), play a key role in inhibiting inflammation and promoting tissue repair (Mantovani et al., 2002).

We provide evidence that cinobufacini may induce the polarization of M1 to M2 macrophages in DSS-induced colitic mice. This finding was confirmed by in vitro experiments using the RAW264.7 macrophage cell line. Notably, cinobufacini promoted a fraction of LPS-induced RAW264.7 cells to polarize into M2 macrophages, while clearly increasing the proportion of CD206 and CD16/32 double-positive cells. Previous studies have also found that CD206 and CD16/32 double-positive cells were increased during the transition from macrophage M1 to M2 (Zhu et al., 2016; Zhang et al., 2018). Our observations of CD206 and CD16/32 doublepositive cells after cinobufacini treatment indicate that cinobufacini may regulate macrophage polarization from M1 to $\mathrm{M} 2$, as we did not observe a large amount of CD206 ${ }^{+} \mathrm{CD} 16 / 32^{-}$ M2 cells during our in vitro experiments.

Regulation of macrophage polarization is one of the most important mechanisms for maintaining immune homeostasis (Formentini et al., 2017). We observed cinobufacini strongly inhibited M1 macrophage polarization in our current study. $\mathrm{NF}-\kappa \mathrm{B}$ p65 is one of the key transcriptional regulators to enhance M1 polarization (Tugal et al., 2013; Kono et al., 2014). Previous studies showed that cinobufacini targeted the NF- $\kappa \mathrm{B}$ pathway to inhibit cancer progression (Wang et al., 2012; Qi et al., 2014). As a canonical stimulus of NF- $\kappa \mathrm{B}$ signaling pathway, LPS binds to Toll-like receptor 4 (TLR4) to activate IKK to phosphorylate $\mathrm{I} \kappa \mathrm{B}$ and liberate NF- $\kappa \mathrm{B}$ (Tugal et al., 2013). In our study, we found cinobufacini reduced the phosphorylation of $\mathrm{I} \kappa \mathrm{B} \alpha$ and $\mathrm{IKK} \alpha / \beta$ protein and inhibited nuclear translocation of $\mathrm{NF}-\kappa \mathrm{B}$ p65 induced by LPS.

Our results suggest that cinobufacini influences phenotypic polarization of macrophages by regulation of NF- $\kappa \mathrm{B}$ activation. We found that cinobufacini also promoted M2 polarization while suppressing M1 polarization. However, the polarization of macrophages to M1 and M2 types is regulated by different signaling pathways. The mechanism by which 
cinobufacini promotes the polarization of M2 macrophages needs to be further studied.

In summary, our study demonstrated that cinobufacini could ameliorate DSS-induced colitis in mice. Cinobufacini inhibits M1 macrophage polarization by suppression of NF- $\kappa \mathrm{B}$ activation (Fig. 8). Our results suggest that cinobufacini deserves further consideration as a potential therapeutic drug for clinical colitis treatment.

\section{Authorship Contributions}

Participated in research design: Wang, Xu, Zhang.

Conducted experiments: Wang, Bai, Weng, Fan, Huang, Zheng.

Performed data analysis: Wang, Bai, Zhang.

Wrote or contributed to the writing of the manuscript: Wang, $\mathrm{Xu}$, Zhang.

\section{References}

Abron JD, Singh NP, Price RL, Nagarkatti M, Nagarkatti PS, and Singh UP (2018) Genistein induces macrophage polarization and systemic cytokine to ameliorate experimental colitis. PLoS One 13:e0199631.

Chassaing B, Aitken JD, Malleshappa M, and Vijay-Kumar M (2014) Dextran sulfate sodium (DSS)-induced colitis in mice. Curr Protoc Immunol 104:Unit 15.25

Eissa N, Hussein H, Kermarrec L, Grover J, Metz-Boutigue ME, Bernstein CN, and Ghia JE (2017) Chromofungin ameliorates the progression of colitis by regulating alternatively activated macrophages. Front Immunol 8:1131.

Feng L, Song P, Zhou H, Li A, Ma Y, Zhang X, Liu H, Xu G, Zhou Y, Wu X, et al. (2014) Pentamethoxyflavanone regulates macrophage polarization and ameliorates sepsis in mice. Biochem Pharmacol 89:109-118.

Formentini L, Santacatterina F, Núñez de Arenas C, Stamatakis K, López-Martínez D, Logan A, Fresno M, Smits R, Murphy MP, and Cuezva JM (2017) Mitochondrial ROS production protects the intestine from inflammation through functional M2 macrophage polarization. Cell Rep 19:1202-1213.

Gan J, Dou Y, Li Y, Wang Z, Wang L, Liu S, Li Q, Yu H, Liu C, Han C, et al. (2018) Producing anti-inflammatory macrophages by nanoparticle-triggered clustering of mannose receptors. Biomaterials 178.95-108.

Jiang X, Yu J, Shi Q, Xiao Y, Wang W, Chen G, Zhao Z, Wang R, Xiao H, Hou C, et al. (2015) Tim-3 promotes intestinal homeostasis in DSS colitis by inhibiting M1 polarization of macrophages. Clin Immunol 160:328-335.

Jin BR, Chung KS, Cheon SY, Lee M, Hwang S, Noh Hwang S, Rhee KJ, and An HJ (2017) Rosmarinic acid suppresses colonic inflammation in dextran sulphate sodium (DSS)-induced mice via dual inhibition of NF- $\kappa$ B and STAT3 activation. Sci Rep 7:46252.

Koh YC, Yang G, Lai CS, Weerawatanakorn M, and Pan MH (2018) Chemopreventive effects of phytochemicals and medicines on M1/M2 polarized macrophage role in inflammation-related diseases. Int J Mol Sci 19:2208.

Kono Y, Kawakami S, Higuchi Y, Maruyama K, Yamashita F, and Hashida M (2014) Antitumor effect of nuclear factor- $\kappa \mathrm{B}$ decoy transfer by mannose-modified bubble lipoplex into macrophages in mouse malignant ascites. Cancer Sci 105:1049-1055.

Kühnemuth B, Mühlberg L, Schipper M, Griesmann H, Neesse A, Milosevic N, Wissniowski T, Buchholz M, Gress TM, and Michl P (2015) CUX1 modulates polarization of tumor-associated macrophages by antagonizing NF- $\kappa \mathrm{B}$ signaling. Oncogene 34:177-187.

Li J, Lei HT, Cao L, Mi YN, Li S, and Cao YX (2018) Crocin alleviates coronary atherosclerosis via inhibiting lipid synthesis and inducing M2 macrophage polarization. Int Immunopharmacol 55:120-127.

Mantovani A, Sozzani S, Locati M, Allavena P, and Sica A (2002) Macrophage polarization: tumor-associated macrophages as a paradigm for polarized M2 mononuclear phagocytes. Trends Immunol 23:549-555.

Mashimo T, Pichumani K, Vemireddy V, Hatanpaa KJ, Singh DK, Sirasanagandla S, Nannepaga S, Piccirillo SG, Kovacs Z, Foong C, et al. (2014) Acetate is a bioenergetic substrate for human glioblastoma and brain metastases. Cell 159: 1603-1614.

Meng Z, Garrett CR, Shen Y, Liu L, Yang P, Huo Y, Zhao Q, Spelman AR, Ng CS, Chang DZ, et al. (2012) Prospective randomised evaluation of traditional Chinese medicine combined with chemotherapy: a randomised phase II study of wild toad extract plus gemcitabine in patients with advanced pancreatic adenocarcinomas. $B r J$ Cancer 107:411-416.

Moore KJ, Sheedy FJ, and Fisher EA (2013) Macrophages in atherosclerosis: a dynamic balance. Nat Rev Immunol 13:709-721.

Okayasu I, Hatakeyama S, Yamada M, Ohkusa T, Inagaki Y, and Nakaya R (1990) A novel method in the induction of reliable experimental acute and chronic ulcerative colitis in mice. Gastroenterology 98:694-702.

Porta C, Rimoldi M, Raes G, Brys L, Ghezzi P, Di Liberto D, Dieli F, Ghisletti S, Natoli G, De Baetselier P, et al. (2009) Tolerance and M2 (alternative) macrophage polarization are related processes orchestrated by p50 nuclear factor $\kappa \mathrm{B}$. Proc Natl Acad Sci USA 106:14978-14983.

Qi J, Tan CK, Hashimi SM, Zulfiker AH, Good D, and Wei MQ (2014) Toad glandular secretions and skin extractions as anti-inflammatory and anticancer agents. Evid Based Complement Alternat Med 2014:312684.

Sica A and Mantovani A (2012) Macrophage plasticity and polarization: in vivo veritas. J Clin Invest 122:787-795

Tugal D, Liao X, and Jain MK (2013) Transcriptional control of macrophage polarization. Arterioscler Thromb Vasc Biol 33:1135-1144.

Viennois E, Tahsin A, and Merlin D (2018) Purification of total RNA from DSStreated murine tissue via lithium chloride precipitation. Bio Protoc 8:e2829.

Wang JY, Chen L, Zheng Z, Wang Q, Guo J, and Xu L (2012) Cinobufocini inhibits $\mathrm{NF}-\kappa \mathrm{B}$ and COX-2 activation induced by TNF- $\alpha$ in lung adenocarcinoma cells. Oncol Rep 27:1619-1624.

Wang L, Xie H, Xu L, Liao Q, Wan S, Yu Z, Lin D, Zhang B, Lv Z, Wu Z, et al. (2017) rSj16 protects against DSS-induced colitis by inhibiting the PPAR- $\alpha$ signaling pathway. Theranostics 7:3446-3460.

Wang SW, Xu Y, Weng YY, Fan XY, Bai YF, Zheng XY, Lou LJ, and Zhang F (2018) Astilbin ameliorates cisplatin-induced nephrotoxicity through reducing oxidative stress and inflammation. Food Chem Toxicol 114:227-236.

Wu X, Zhao H, Wang H, Gao B, Yang J, Si N, and Bian B (2012) Simultaneous determination of eight bufadienolides in cinobufacini injection by HPLC coupled with triple quadrupole mass spectrometry. J Sep Sci 35:1893-1898.

Wynn TA and Vannella KM (2016) Macrophages in tissue repair, regeneration, and fibrosis. Immunity 44:450-462.

Xie S, Spelmink L, Codemo M, Subramanian K, Pütsep K, Henriques-Normark B, and Olliver M (2016) Cinobufagin modulates human innate immune responses and triggers antibacterial activity. PLoS One 11:e0160734.

Zhang D, Zheng J, Ni M, Wu J, Wang K, Duan X, Zhang X, and Zhang B (2017a) Comparative efficacy and safety of Chinese herbal injections combined with the FOLFOX regimen for treating gastric cancer in China: a network meta-analysis. Oncotarget 8:68873-68889.

Zhang J, Dou W, Zhang E, Sun A, Ding L, Wei X, Chou G, Mani S, and Wang Z (2014) Paeoniflorin abrogates DSS-induced colitis via a TLR4-dependent pathway. Am J Physiol Gastrointest Liver Physiol 306:G27-G36.

Zhang X, Xu F, Liu L, Feng L, Wu X, Shen Y, Sun Y, Wu X, and Xu Q (2017b) (+)-Borneol improves the efficacy of edaravone against DSS-induced colitis by promoting M2 macrophages polarization via JAK2-STAT3 signaling pathway. Int Immunopharmacol 53:1-10.

Zhang Y, Feng J, Fu H, Liu C, Yu Z, Sun Y, She X, Li P, Zhao C, Liu Y, et al. (2018) Coagulation factor X regulated by CASC2c recruited macrophages and induced M2 polarization in glioblastoma multiforme. Front Immunol 9:1557.

Zhu W, Jin Z, Yu J, Liang J, Yang Q, Li F, Shi X, Zhu X, and Zhang X (2016) Baicalin ameliorates experimental inflammatory bowel disease through polarization of macrophages to an M2 phenotype. Int Immunopharmacol 35:119-126.

Address correspondence to: Dr. Feng Zhang, Department of Central Laboratory, People's Hospital of Quzhou, 2 Zhongloudi Road, Quzhou, 324000, People's Republic of China. E-mail: felix.f.zhang@outlook.com; or Yi $\mathrm{Xu}$, Department of Urology, People's Hospital of Quzhou, 2 Zhongloudi Road, Quzhou, 324000, People's Republic of China. E-mail: yixumd@126.com 